\title{
Performance Analysis of Micro blaze Implemented GPS-INS Integrated Systems on Spartan 6 and Zynq FPGAs
}

\author{
B.LokeswaraRao, Associate Professor, Dr.K.V.V.SReddy, Former Professor, \\ Dr.G.Sasi Bhushana Rao, Professor\& HOD \\ Dept. of ECE, BIET, Hyderabad, A.P, INDIA \\ Dept. of ECE, Andhra University College of Engineering (A), Visakhapatnam, A.P, INDIA \\ Dept. of ECE, Andhra University College of Engineering (A), Visakhapatnam, A.P, INDIA
}

\begin{abstract}
This paper compares the performances of two loosely coupled Micro blaze Implemented GPS-INS Integrated Systems On Spartan 6 And Zynq FPGAs. The first system usesa Micro blaze implemented GPS-INS integrated system on Spartan 6 Field Programmable Gate Array (FPGA) for inertial navigation solution and Kalman filter computation.The second system uses a Micro blaze implemented GPS-INS integrated system on Zynq FPGA for inertial navigation solution and Kalman filter computation. Real time issues related to accuracy of position, GPS outages, Selective availability of GPS, Higher variances of accelerometers, Resource usage of FPGA in terms of Slices, DSP48 and Block Random Access Memory (BRAM), Computation time, latency, and power consumption are presented.The systems are designed to give real time processed navigation solutions with an update rate of $100 \mathrm{~Hz}$.

Key words: Global positioning system, Inertial navigation system, Kalman filter,Spartan 6 FPGA,Zynq FPGA.
\end{abstract}

\section{Introduction}

MEMS-based Inertial Measurement Unit(IMU) systems have proved to be highly popular and feasible for autonomous navigation systems[1-9].These systems are cost effective,compact and light weight.They do not have good precision.

A loosely coupled integrated approach $[1,4]$ using a GPS receiver and alow cost strap down INS, is used to overcome the sensor errors or random disturbances.Many papers have reported the processing of the GPS INS signalsoffline[7]. The inertial navigation computation is performed at $100 \mathrm{~Hz}$.Thisinertial datais then integrated with the GPS data at $1 \mathrm{~Hz}[10]$.

Coupling of low cost inertial sensors with GPS is broadly classified as Loosely coupled system , Tightly coupled system and Ultra-tightly coupled system.

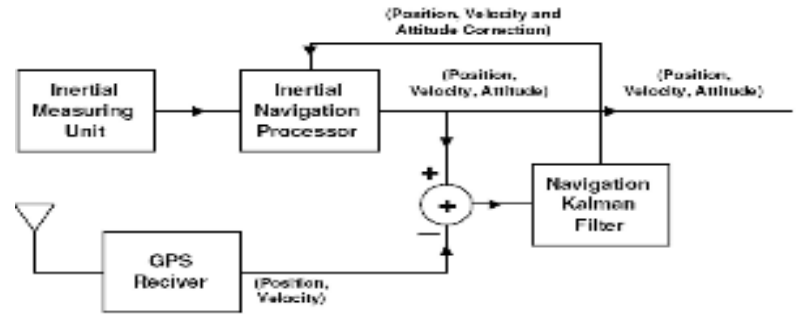

Fig.1.Loosely Coupled Integrated GPS-INS System

a) Loosely coupled system: In this system GPS

data, i.e., position, velocity, etc. are coupled with INS data using an Extended Kalman Filter( EKF) as shown in Fig. 1. It is highly dependent on the availability of GPS data. In the present work, a nine state Kalman filter is used.

b) Tightly coupled system: In this system, the

INS data is directly fused with GPS raw measurement data inside the GPS Kalman filter as shown in Fig.2. It is more robust compared with the loosely coupled system. However, it is also more complex.

c) Ultra-tightly coupled system

This type of integration method requires access to the receiver's firmware. As a result, this scheme of integration is usually implemented only by the equipment manufacturer. 


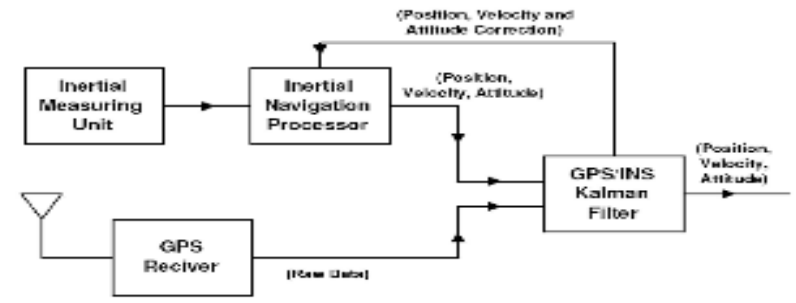

Fig.2. Tightly Coupled Integrated GPS-INS System

An integrated system based on low cost Inertial Measurement Unit(IMU) and GPS receiver has been proposed by Moon et al[4].Faulkner et al.,[5] have described a development program in which a PC/104 computer is used for loosely coupled system and digital signal processor(DSP) for developing the closely(tightly) coupled integrated system. The system developed by Hegg[6] uses six different power supplies for their integrated scheme.Bridging GPS outages for tens of seconds using a low cost inertial device, and integration with GPS information and its feasibility are described by Cao et al.,[7,8].An improved design and fabrication of a loosely coupled INS/GPS integrated system for compact and low powerapplications has been proposed by Agarwal et al.,[11].

Details of the of Micro blaze implemented GPS/INS Integrated System on Spartan 6 and Zynq FPGAs suitable for autonomous navigation systems are presented. It may be noted that the emphasis is on real time issues related to accuracy of position, GPS outages, Selective availability of GPS, Higher variances of accelerometers, resource usage of FPGA in terms of slices,DSP48 and BRAM, Computation time, latency, and power consumption . A loosely coupled integrated approach [1,4] is chosen to overcome the sensor errors and obtain accurate estimates of position and attitude.

The remaining part of this paper is organized as follows. System architecture of the proposed systems is described in Section II. In Section III, Micro blaze implementation on Spartan 6 and Zynq FPGAs are presented. Results and discussions are included in Section IV, followed by conclusions of this work in Section $\mathrm{V}$.

\section{Proposed System Architecture}

The proposed integrated systems providing the navigation system function are shown in Fig. 3 and Fig.4. For better understanding, the system can be divided into four main blocks as INS Module, GPS Module, Sensor Modelling and Kalman Filter Module The proposed architecture is now explained in details.

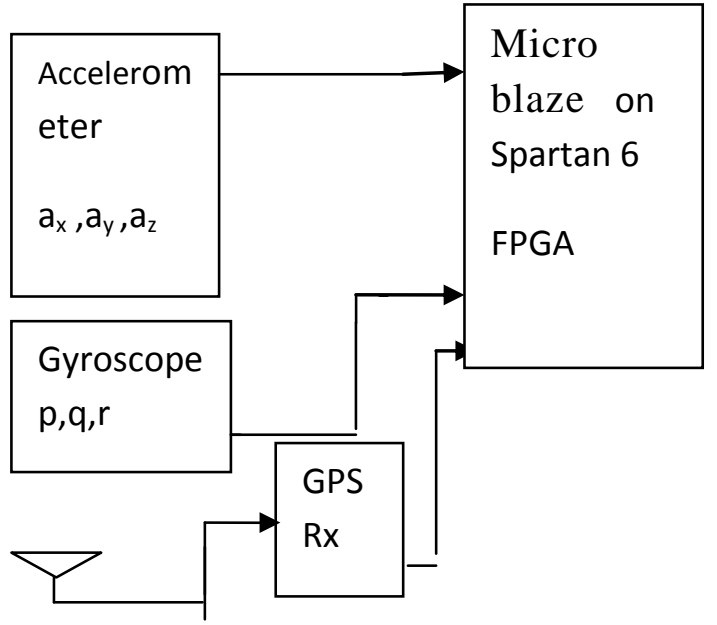

Fig.3.Proposed System Architecture

A program called Flight Dynamics and Controls(FDC ) toolbox, when given the initial conditions of the aircraft thrust and aerodynamics, gave as its output the time history of the aircraft in the form of a state vector $X$, where

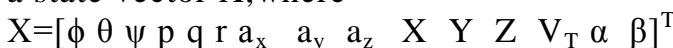

$-\phi \theta \psi$ are the Euler angles in radians,

- $\mathrm{p} \mathrm{q} \mathrm{r}$ are the roll,pitch and yaw rates from the gyroscopes in radians per second, 
- $a_{x} \quad a_{y} \quad a_{z}$ are the accelerations from the accelorometers in $\mathrm{m} / \mathrm{s}^{2}$,

- $\mathrm{X} \mathrm{Y} \mathrm{Z} \mathrm{are} \mathrm{the} \mathrm{distances} \mathrm{along} \mathrm{the} \mathrm{three} \mathrm{axes} \mathrm{in} \mathrm{the} \mathrm{navigation} \mathrm{frame} \mathrm{in} \mathrm{meters,}$

$-\mathrm{V}_{\mathrm{T}} \alpha \beta$ are the velocity of the aircraft in $\mathrm{m} / \mathrm{s}$, the angle of attack in radians and the sideslip angle in radians, respectively.

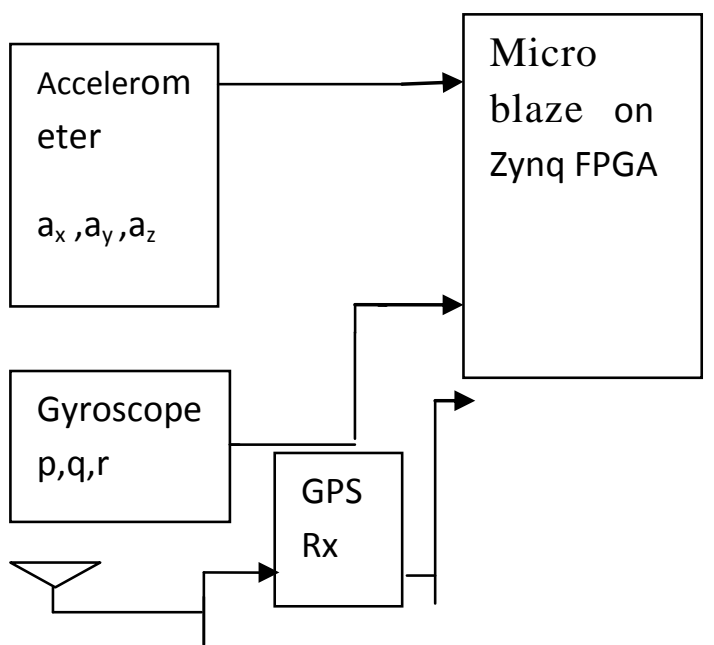

Fig.4.Proposed System Architecture

The FDC program can generate these values at any time step as required.Typical time steps or update rates range from $10 \mathrm{~ms}-100 \mathrm{~ms}$.

\subsection{INS Module}

The INS program now takes 6 states from this time history,viz.p,q,r, $a_{x}, a_{y}, a_{z}$. These act as if the program is reading directly from the gyros and accelerometers. Then the program integrates and calculates four Euler parameters.From these Euler parameters, the Euler angles are calculated.Now the accelerations from the accelerometers are used to get the values of $\mathrm{U}, \mathrm{V}, \mathrm{W}$.

We now have the velocity components of the aircraft in the body frame.To convert it to the navigation frame or local earth frame, we use the DCM matrix and calculate $\mathrm{V}_{\mathrm{T}}$.

These velocity components are then integrated to get the position $\mathrm{X}, \mathrm{Y}, \mathrm{Z}$ along the three axes in the local earth frame.The latitude,longitude and height can be calculated.All the integrations are carried out using the fourth order Runge-Kutta methods.The INS Module is shown in Fig.5.

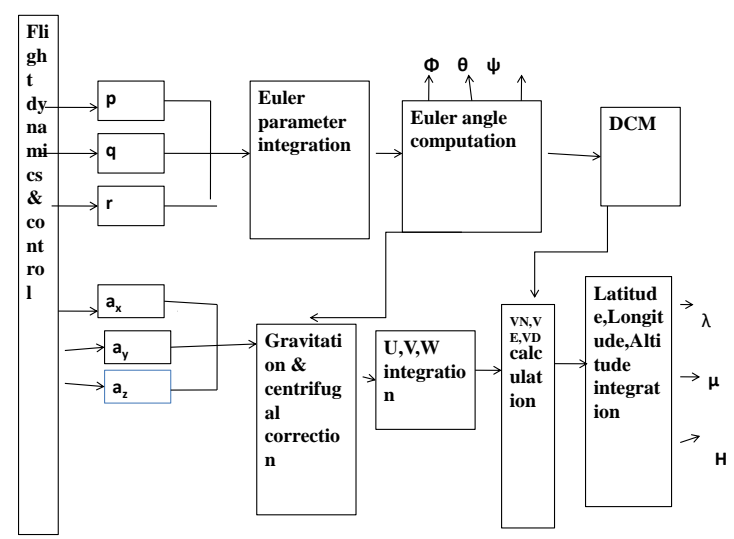

Fig.5.INS Module

\subsection{GPS Module}

The GPS gives the latitude,longitude and altitude of the current location the receiver. What our program does is that it converts the $\mathrm{X}, \mathrm{Y}, \mathrm{Z}$ given out by the FDC into latitude, longitude and altitude as would be given out by the GPS receiver.The update rate is 1 second.The GPS program uses WGS - 84 approximatio in which the earth is considered as an ellipse with a semi-major axis(equatorial radius) of $a=6,378,137 \mathrm{~m}$, and a semi-minor axis(polar radius) of $b=6,356,752.3142 \mathrm{~m}$. 
It is necessary to define the distance corresponding to a $1^{\circ}$ change in longitude $\left(\mathrm{F}_{\text {lon }}\right)$ and latitude $\left(\mathrm{F}_{\text {lat }}\right.$ ) for a specified location(latitude and height or altitude).

Hence, the latitude and longitude at the current $\operatorname{location}\left(\lambda_{2}, \mu_{2}\right)$ can be calculated from the latitude and longitude from the previous $\operatorname{location}\left(\lambda_{1}, \mu_{1}\right)$.If we consider the earth as a sphere, $F_{\text {lon }}$ and $F_{\text {lat }}$ can be replaced by just the radius of the earth and the latitude and longitude can be calculated.However, to make the GPS modeling more authentic, we have considered the earth as an ellipse.The GPS

Module is shown in Fig.6.

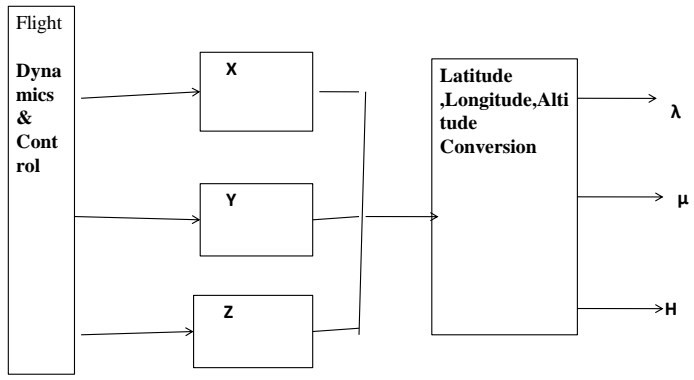

Fig.6.GPS Module

\subsection{Sensor Modelling}

The accelerometer senses the acceleration in terms of $\mathrm{g}$ and sends it to the INS in Volts by conversion using a scale factor.A certain offset at zero $g$ called the bias exists by default. The scale factor and the bias details are available from the specification sheets of the accelerometers.Errors arise in the acceleration sensed because the scale factor and the bias are not fixed[11].They vary stochastically and they lie within a certain range which is specified in the data sheets of the accelerometers.

The gyroscope error modelling is also done in a similar way accounting for the corresponding scale factors and offset biases[11].These errors together lead to drift,which grows with time in the output(location) given by the INS and it could be up to hundreds of meters. Table I gives a set of values given by the specification sheets which were used in the simulation[14-16].The errors due to temperature effects and due to the misalignment of accelerometers and gyroscopes have been ignored.

TABLE I

Sensor Specifications used in Simulation

\begin{tabular}{|l|l|l|}
\hline Quality & Vlaue & Standard Deviation \\
\hline $\begin{array}{l}\text { Scale factor of the } \\
\text { accelerometer }\end{array}$ & $250 \mathrm{mV} / \mathrm{g}$ & $\pm 25 / 3 \mathrm{mV} / \mathrm{g}$ \\
\hline $\begin{array}{l}\text { Zero g Offset of the } \\
\text { gyroscope }\end{array}$ & $2500 \mathrm{mV}$ & $\pm 625 / 3 \mathrm{mV}$ \\
\hline $\begin{array}{l}\text { Scale factor of the } \\
\text { gyroscope }\end{array}$ & $1.11 \mathrm{mV} / 0 / \mathrm{s}$ & $\pm 10 / 3 \%$ \\
\hline $\begin{array}{l}\text { Typical turn-on } \\
\text { drift of the } \\
\text { gyroscope }\end{array}$ & $0.12 \% / \mathrm{s}$ & - \\
\hline $\begin{array}{l}\text { Random noise } \\
\text { incorporated in the } \\
\text { GPS }\end{array}$ & - & $\pm 20 \mathrm{~m}$ \\
\hline
\end{tabular}

\subsection{Kalman Filter Module}

The error dynamics model given in the works of Schmidt[12],Bar-Itzhack et al[13],Grewal[1] has been used for simulation. The error dynamics equations are obtained when the nominal equations are perturbed in the local level north-pointing coordinate system that corresponds to the geographic location indicated by the INS.The differential equations that describe the error behavior of the INS are devided into equations describing the propagation of the translatory errors and equations describing the propagation of attitude errors. The translatory and the attitude errors are not coupled to each 
other.The nine state INS/GPS integration Kalman filter will then be built using the error dynamics equations.The Kalman Filter Module is shown in Fig.7.

The Micro blaze implementation of the prototype integrated GPS/INS systems on Spartan 6 and Zynq FPGAs are discussed in detail in the next section.

ERROR IMPLEMENTATION

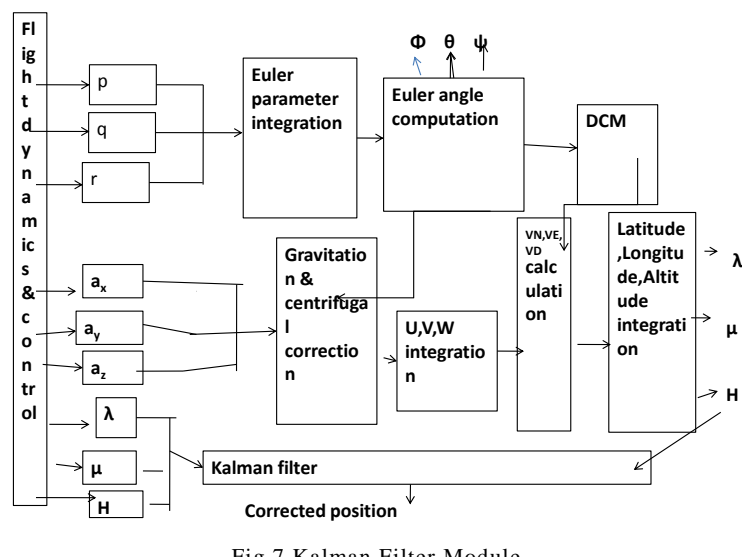

III. Implementation With Micro Blaze

This section describes about the Micro blaze implemented GPS-INS integrated systems on Spartan 6 and ZynqFPGAs .

\subsection{Micro blaze implementation on Spartan 6 FPGA}

The GPS/INS integration with Kalman filter implemented on Spartan-6 XC6SLX45T-3FGG484 FPGA reconfigurable hardware is shown in Fig.8[17]. The choice of reconfigurable hardware is based on the envisaged applications of this GPS/INS integration system in military and high speed avionics. The SP605 board from Xilinx is used as target hardware for hardware level verification.

The hardware software co-design flow with Xilinx's Microblaze softprocessor core is adopted here.

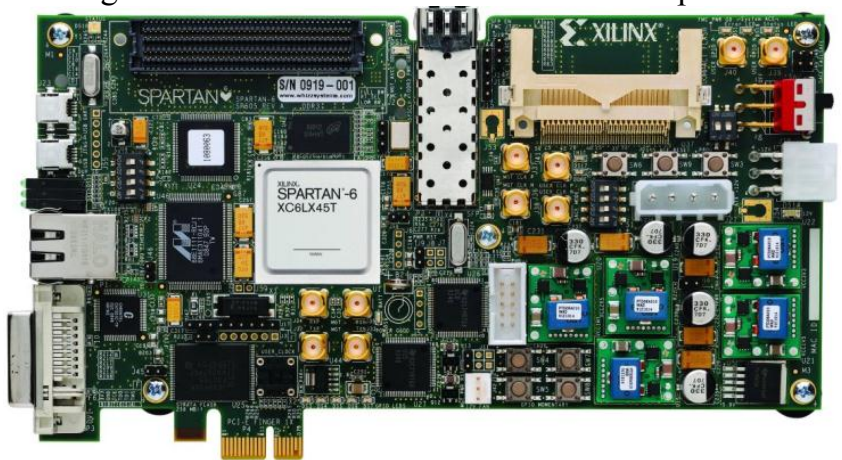

Fig.8. SP605 board

The INS data server, GPS data server are implemented as hardware Intellectual Property(IP) cores provide the data samples to the Microblaze softprocessor. The Kalam filtering algorithm is implemented in $\mathrm{C}$ and ported to Microblaze along with its board support files. The IEEE 754 single precesion floating point unit (FPU) core is added to microblaze as a peripheral unit. The entire Processor Local Bus(PLB) based hardware system configuration using Xilinx Platform Studio is shown in Fig.9. 


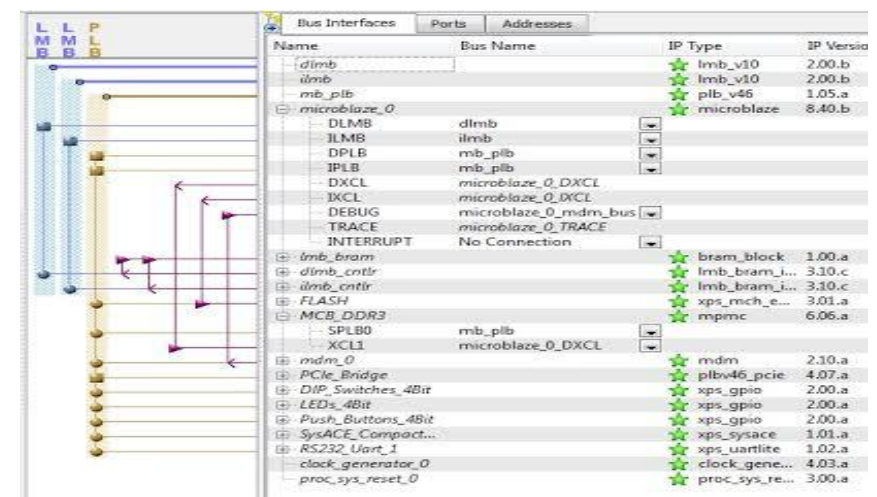

Fig.9. Hardware plat form with Micro blaze and peripherals

The USB-UART available on board is used for reading the algorithm inputs and outputs to personal computer (PC) for further analysis. The onboard JTAG debugger is used for configuration and runtime debugging.

MATLAB is used for analyzing the logged data and results are presented in next section.

\subsection{Micro blaze implementation onZynq FPGA}

The GPS/INS integration with Kalman filter implemented on Zynq-7000 XC7Z020-CLG484-1 SOC hardware is shown in Fig.10[18]. The choice of reconfigurable hardware is based on the envisaged applications of this GPS/INS integration system in military and high speed avionics. The Zed board from Xilinx is used as target hardware for hardware level verification.

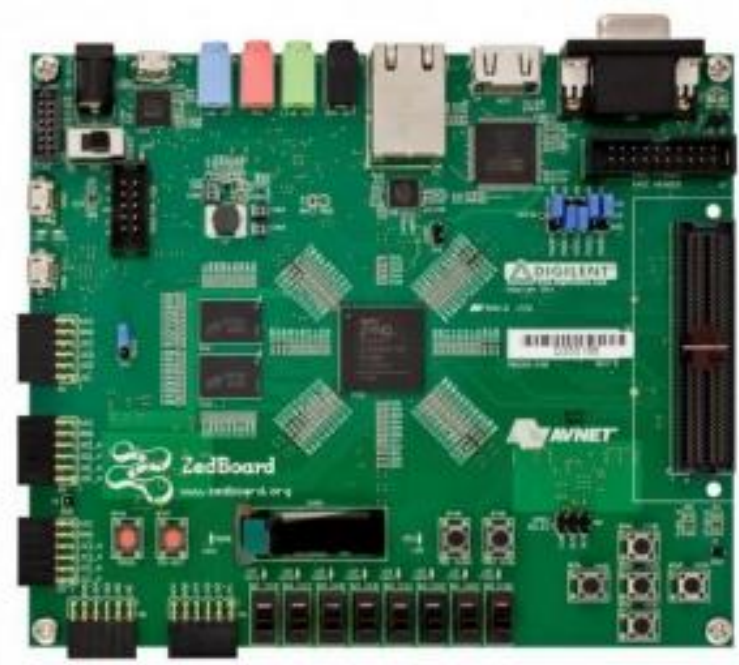

Fig.10. Zedboard with Zynq 7 series FPGA

The block diagram of the Zed board with Zynq 7 series FPGA is shown in Fig.11 [18].

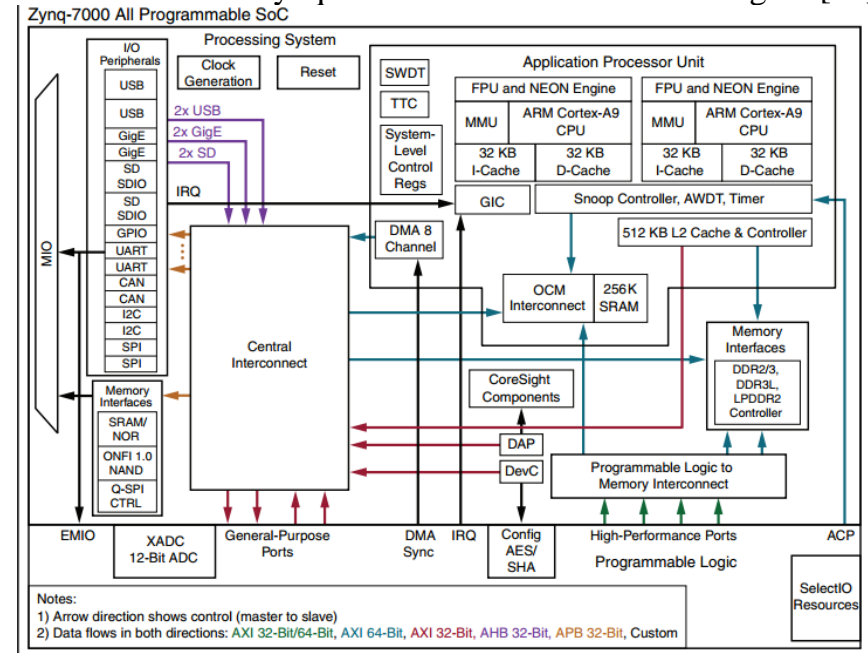

Fig.11. Block diagram of Zynq 7 series SOC 
The hardware software co-design flow with Xilinx's Zynq's ARM-9 hardcore processor core is adopted here. The INS data server, GPS data server are implemented as hardware IP cores provide the data samples to the ARM-9 processor. The Kalman filtering algorithm is implemented in $\mathrm{C}$ and ported to ARM-9 along with its board support files. The IEEE 754 single precesion floating point unit (FPU) core is enabled. The entire AXI bus based hardware system configure using Xilinx Platform Studio is shown in Fig.12[17].

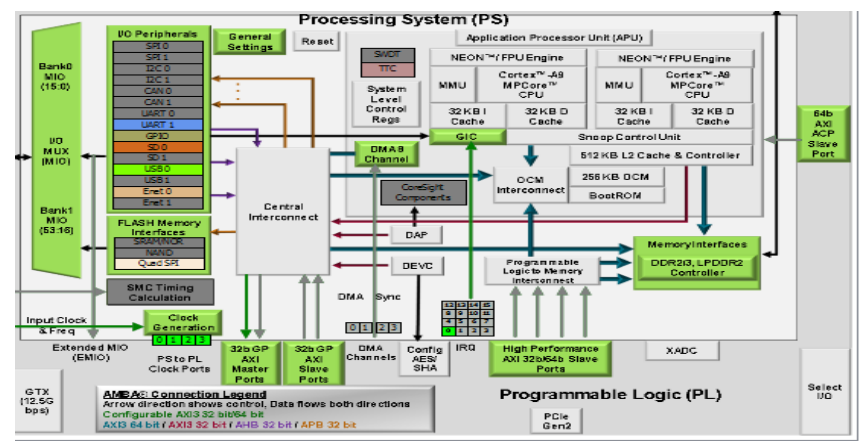

Fig.12.Hardware platform with ZYNQ and peripherals

The USB-UART available on board is used for reading the algorithm inputs and outputs to personal computer (PC) for further analysis. The onboard JTAG debugger is used for configuration and runtime debugging. MATLAB is used for analyzing the logged data and results are presented in next section.

\section{Results And Discussions}

Input for the system is read from the data stored on the hard drive and the program is run just as if the collection (of data) was taking place in real-time. The simulated sensor data are generated using trajectories obtained from flight dynamic and control tool box (FDC) in MATLAB. Aircraft states were stored to simulate sensors. To analyze the prediction accuracy of INS, it was compared with a true trajectory generated using MATLAB. Simulated results include various errors in inertial sensors and the GPS. Same sensor outputs were given to the present Micro blaze implementation on Spartan 6 FPGA and the results were compared. The output waveforms obtained from the unaided INS, GPS and actual trajectory are shown in Figs.13-15. The output waveforms obtained from the integrated GPS-INS system, the GPS, , and the actual trajectory are shown in Figs.15-17. Trajectory plots showing usage of higher variance of accelerometers are shown in Figs.18-20. Trajectory plots showing a GPS "outage", are shown in Figs.21-23. Trajectory plots showing Selective Availability of GPS are shown in Figs.24-26. The plots for latitude, longitude, and altitude obtained directly from the hardware (Micro blaze implemented on Spartan 6 FPGA output) are shown in Figs. 27-29. The plots for latitude, longitude, and altitude obtained directly from the hardware (Micro blaze implemented on Zynq FPGA output) are also the same as the plots shown in Figs. 27-29.They show good agreement with the actual trajectory.

The resource utiliation report of developed system on Spartan-6 FPGA are given in Table II. As the area consuming algorithm blocks are implemented on Microblaze and performance demanding blocks are implemented on FPGA fabrics, the implemented system demonstrates optimal area occupancy and high speed implementation.

\section{TABLE II}

Resource Utiliation

\begin{tabular}{|l|l|l|l|}
\hline Resource & Total available & Used & $\%$ utilization \\
\hline Slice Registers & 54,576 & 7,293 & $13 \%$ \\
\hline Slice LUTs & 27,288 & 8,429 & $30 \%$ \\
\hline BRAM & 116 & 31 & $21 \%$ \\
\hline DSP48 & 58 & 8 & $13 \%$ \\
\hline
\end{tabular}

The maximum clock speed achieved by Microblaze softprocessor is $125 \mathrm{MHz}$. The present system is tested at $88 \mathrm{MHz}$ clock speed. The FPGA running total integration system is profiled for speed and latencies by running for 900 seconds of data.The Kalman filter iterations are presently being computed at $10 \mathrm{~ms}$ interval. However the implemented logic is able to achieve the latency of $0.9 \mathrm{~ms}$ for every iteration. Hence the present system can integrate with INS system with update rate of $1 \mathrm{~ms}$. The resource utiliation report of developed system on Zynq SOC FPGA is given in Table III. As the area consuming algorithm blocks are implemented on ARM-9 Cortex and performance demanding 
blocks are implemented on FPGA fabrics, the implemented system demonstrates optimal area occupancy and high speed implementation. Note that as the ARM-9 Cortex has hard core processor, no BRAM and DSP48 slices will be consumed from FPGA fabrics.

TABLE III

Resource Utiliation

\begin{tabular}{|l|l|l|l|}
\hline Resource & Total available & Used & $\%$ utilization \\
\hline Slice Registers & 106,400 & 2080 & $2 \%$ \\
\hline Slice LUTs & 53,200 & 4,159 & $8 \%$ \\
\hline BRAM & 140 & 0 & $0 \%$ \\
\hline DSP48 & 220 & 0 & $0 \%$ \\
\hline
\end{tabular}

The maximum clock speed achieved by RTL logic is $151 \mathrm{MHz}$. The FPGA running total integration system is profiled for speed and latencies by running for 900 seconds of data.

The Kalman filter iterations are presently being computed at $10 \mathrm{~ms}$ interval. However the implemented logic is able to achieve the latency of $0.41 \mathrm{~ms}$ for every iteration. Hence even the present system can integrate with INS system with update rate of $0.5 \mathrm{~ms}$.

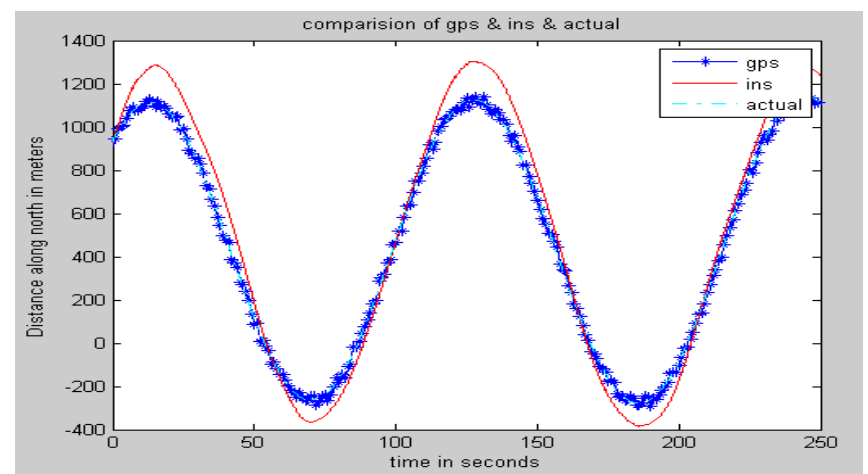

Fig. 13. Distance along North calculated by unaided INS and GPS

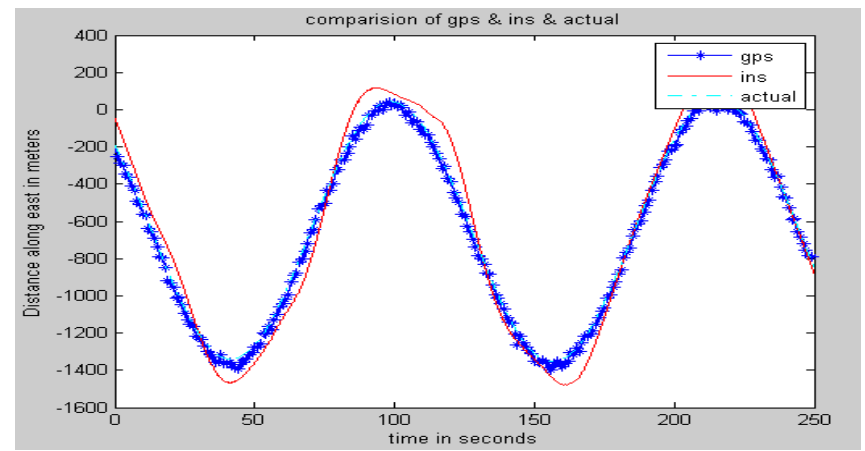

Fig. 14. Distance along East calculated by unaided INS and GPS

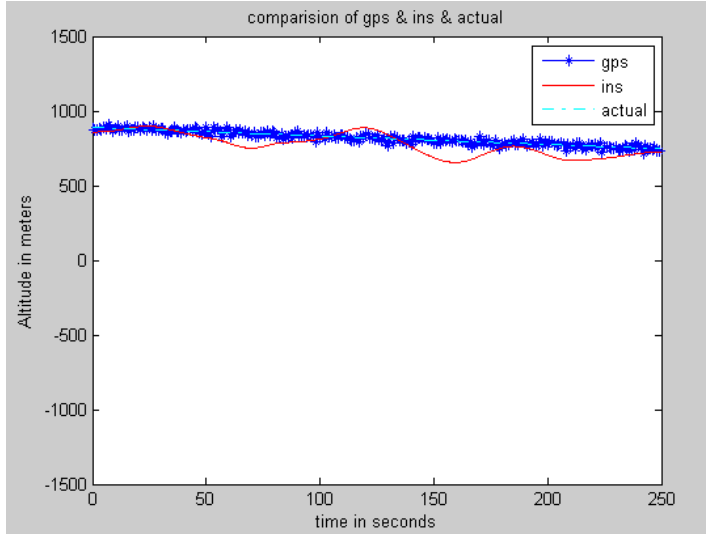

Fig. 15. Altitude along East calculated by unaided INS and GPS 


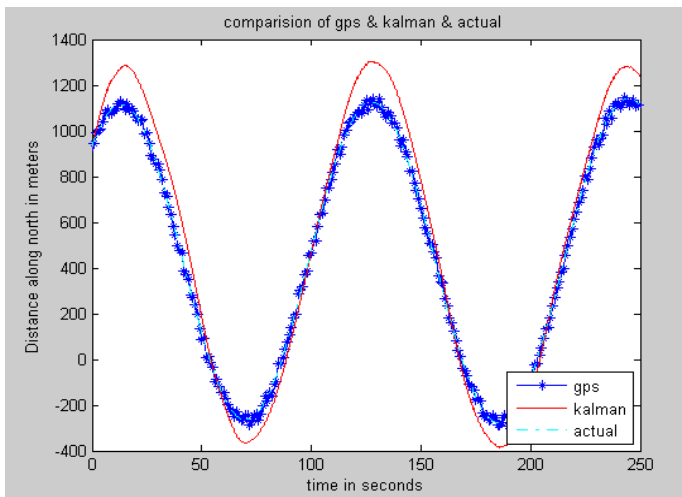

Fig. 16.Kalman filtered output of distance along North

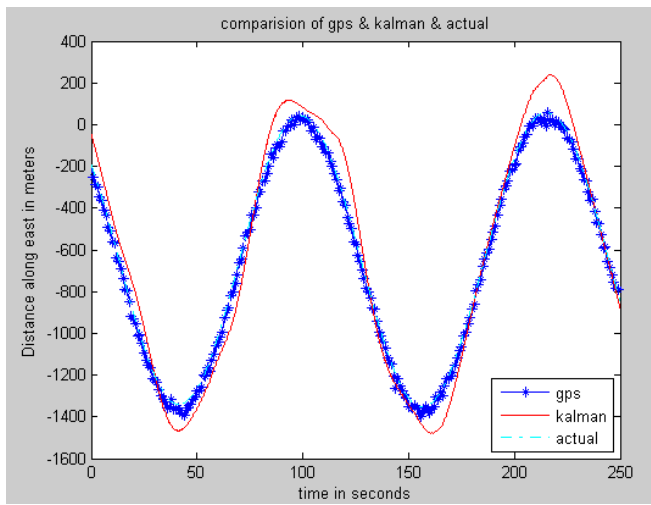

Fig. 17.Kalman filtered output of distance along East

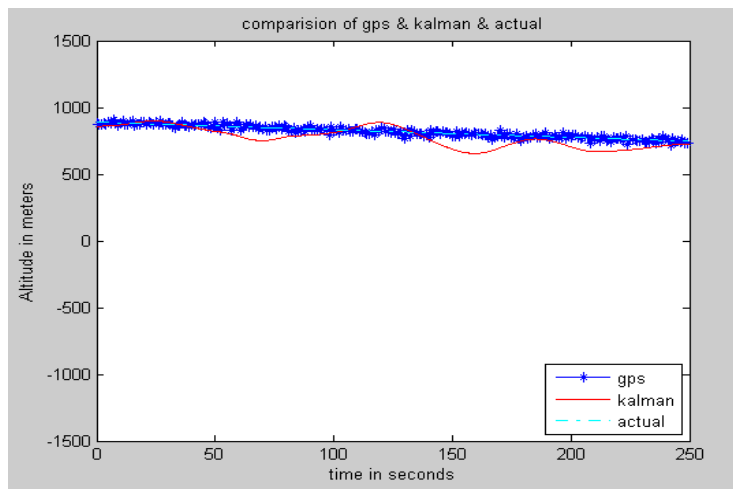

Fig. 18.Kalman filtered output of Altitude

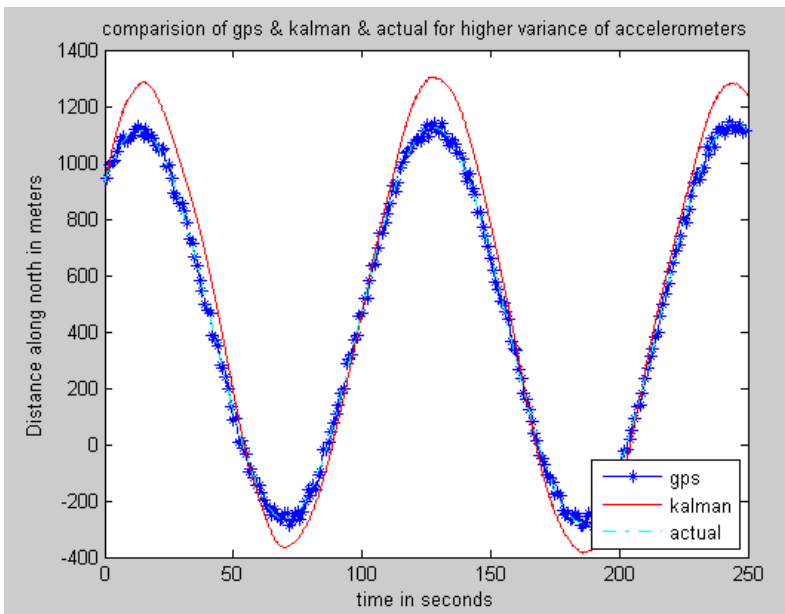

Fig. 19. Distance along North calculated with higher variances of accelerometers 


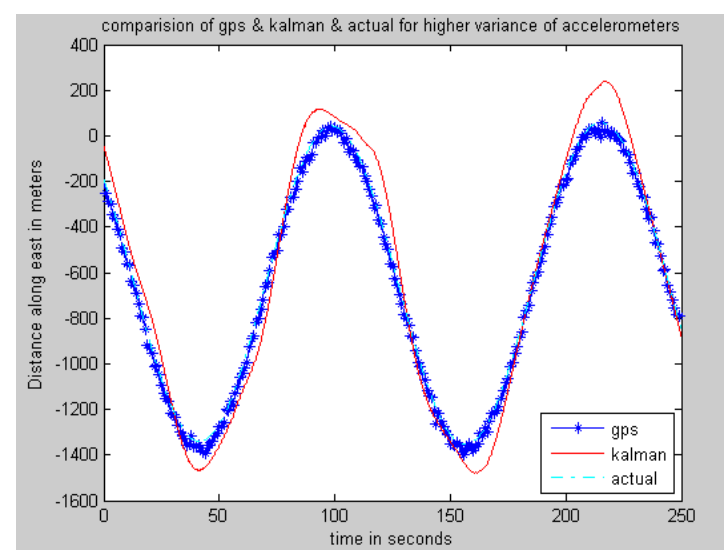

Fig.20. Distance along East calculated with higher variances of accelerometers

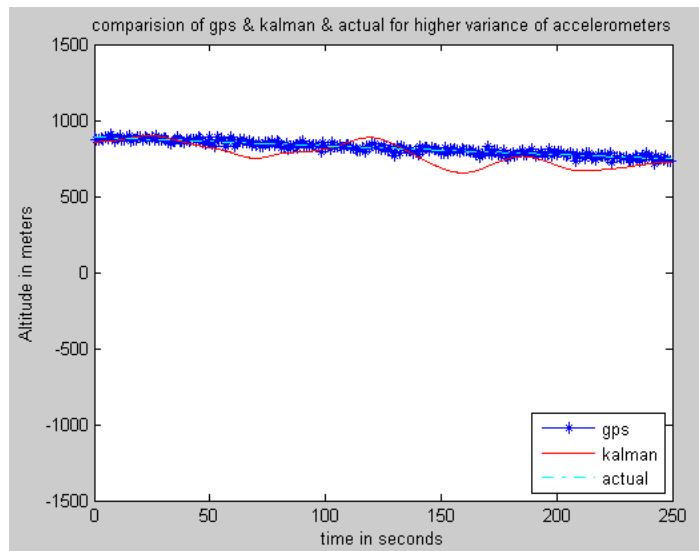

Fig.21. Altitude calculated with higher variances of accelerometers

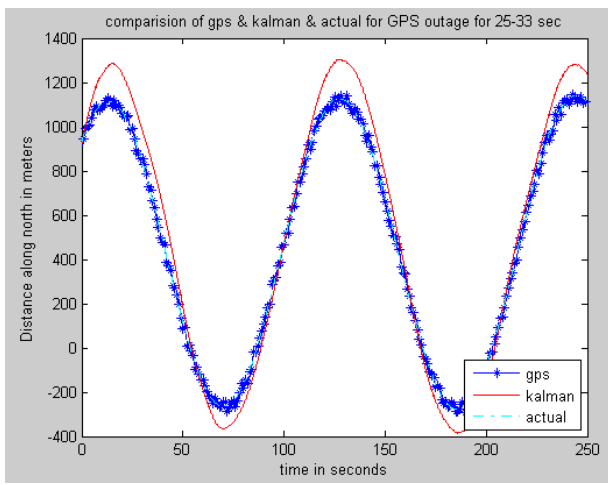

Fig.22. Distance along North calculated with GPS outage between 25s and 33s

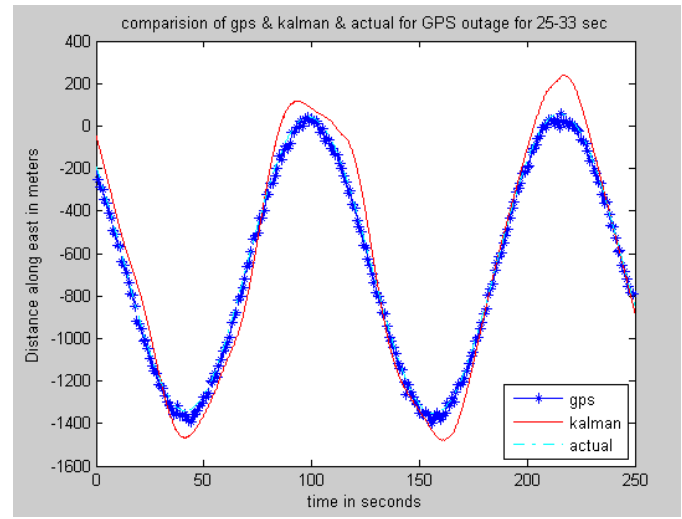

Fig. 23. Distance along East calculated with GPS outage between 25s and 33s 


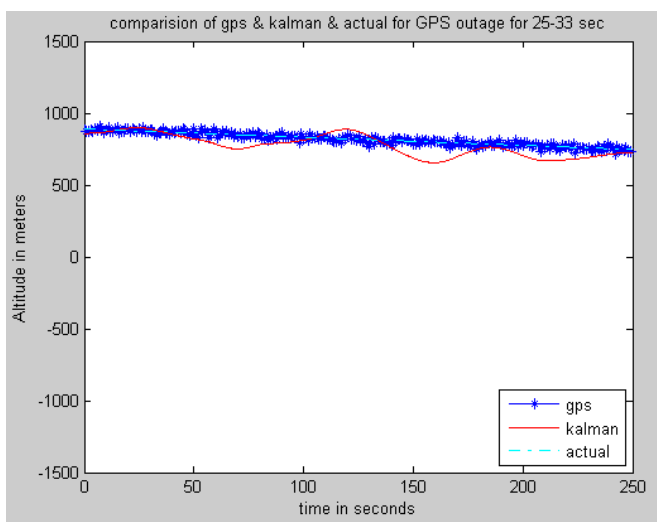

Fig. 24.Attitude calculated with GPS outage between $25 \mathrm{~s}$ and $33 \mathrm{~s}$

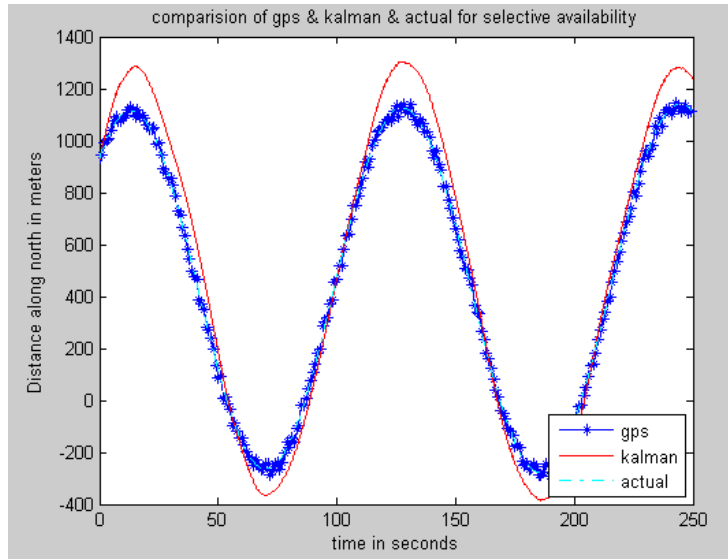

Fig. 25. Distance along North calculated with Selective availability introduced

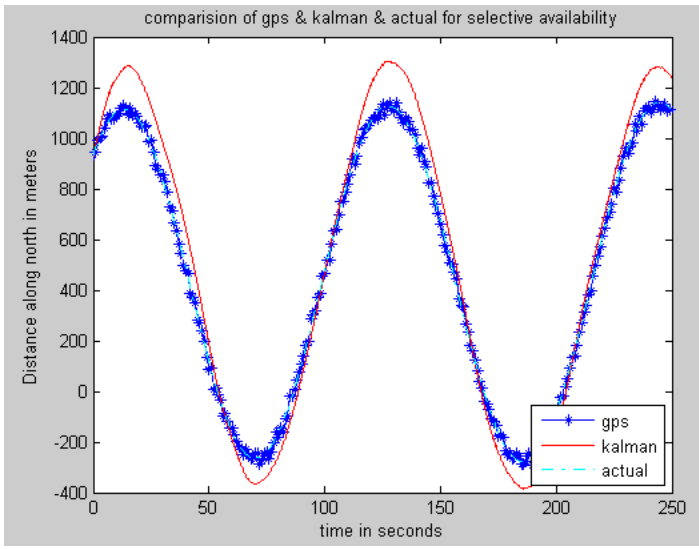

Fig. 26. Distance along East calculated with Selective availability introduced

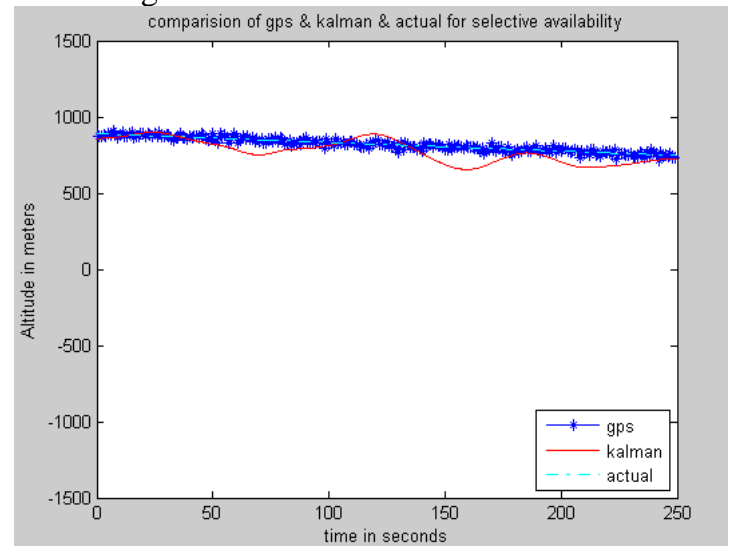

Fig. 27. Attitude calculated with Selective availability introduced 


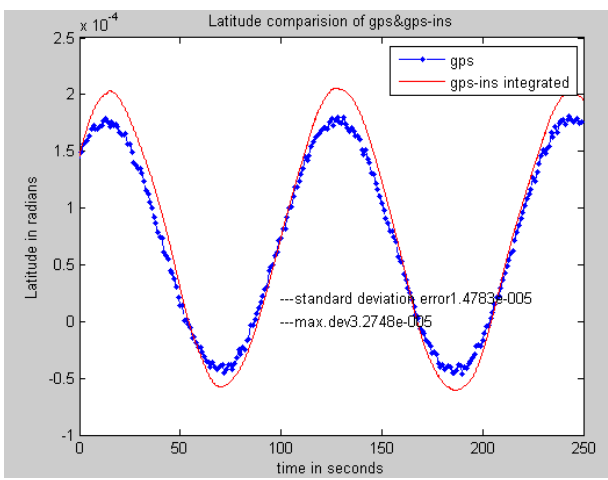

Fig. 28.Spartan 6 FPGA (hardware) output.Latitude versus time.

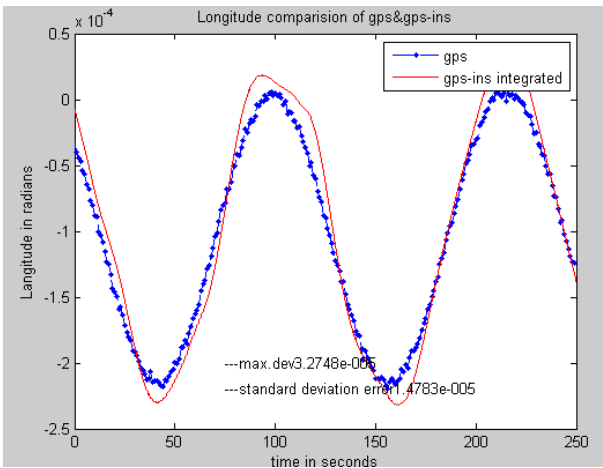

Fig.. 29. Spartan 6 FPGA (hardware) output. Longitude versus time. .

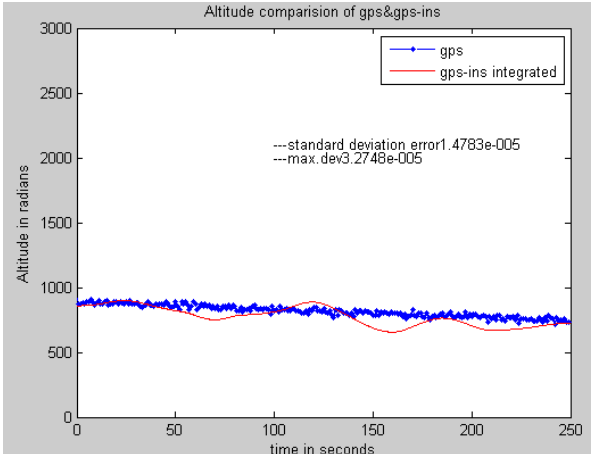

Figure. 27. Spartan 6 FPGA (hardware) output. Altitude versus time.

\section{Conclusions}

The paper discussed a new and a better approach to fuse the data from the GPS and INS using Kalman filter . From the test results over a span of $250 \mathrm{~s}$, it is observed that after initialization, the position accuracy of INSGPS system is comparable to that of the GPS receiver. The unaided INS ( red line) deviates from the actual trajectory (green line) by a small extent. It must be noted that the output of the Kalman filter is bounded by the GPS output. Trajectory plots showing a GPS "outage" and its effect are shown. Trajectory plots showing a much better accuracy by using higher variance of accelerometers are shown. Trajectory plots showing Selective Availability of GPS and its effect are shown.

The implemented system with hardware software co-design approach on Spartan 6 FPGA occupies only $30 \%$ of slices and $13 \%$ of DSP48 resources, with maximum achivable clock speed of $125 \mathrm{MHz}$. The latency for one iteration of Kalman filter is less than $1 \mathrm{~ms}$, hence suitable for integrating with high speed INS units. The Spartan -6 FPGA consumes $173 \mathrm{~mW}$ of power.

The implemented system with hardware software co-design approach on Zynq SOC FPGA occupies only $8 \%$ of slices, with maximum achivable clock speed of $151 \mathrm{MHz}$. The latency for one iteration of Kalman filter is less than $0.5 \mathrm{~ms}$, hence suitable for integrating with high speed INS units. The Zynq-7 SOC FPGA consumes $82 \mathrm{~mW}$ of power.

The GPS/INS integration along with Kalman filter implemented on both Spartan-6 and Zynq-7 FPGAs shows promising results by using reconfigurable hardware software codesign approach for future naviation systems. The Zynq-7 SOC FPGA, consisting ARM-9 Cortex core in it, demonstrates twice 
the higher speed while consuming only half of the power in comparison with spartan-6 FPGA. The Zynq based implementation also proven to be area efficient by occupying only $8 \%$ of resouces in comparison with Spartan-6 implemenation occupying 30\% of resouces.

However the spartan-6 FPGA softcore implementation creates opportunity for multicore instantiation and hence parallel Kalman filter execution for even complex GPS/INS integration algorithms.

\section{References}

[1] Grewal, M. S., Weill, L. R., and Andrews, A. P. Global Positioning Systems. Inertial Navigation, and Integration. New York: Wiley, 2001.

[2] Sukkarieh, S., Nebot, E. M., and Durrant-Whyte, H. F. A high integrity IMU/GPS navigation loop for autonomousland vehicle applications. IEEE Transactions on Robotics and Automation, 15, 3 (June 1999), 572 - 578

[3] Shang, J., Mao, G., and Gu, Q. T.Design and implementation of MIMU/GPS integrated navigation systems.In IEEE Position Location and Navigation Symposium,Apr. 2002, 99-105.

[4] Moon, S. W., Hwang, D. H., Sung, T. K., and Lee, S. J. Design and Implementation of an Efficient Loosely-Coupled GPS/INS integration scheme.Technical Report, Chungnam National University, Korea, 1998.

[5] Faulkner,N.M.,Cooper, S,J., and Jeary, P.A.Integrated MEMS/GPS navigation systems, In IEEE Position Location and Navigation Symposium April 2002, 306-313.

[6] Hegg.J.Enhanced space integrated GPS/INS(SIGI)IEEE Aerospace and Electronic systems Magazine, 17, 4. April 2002, $26-33$.

[7] Cao, F.X., Yang, D.K., Xu, A.G., Ma, J., Xiao, W.D., Law, C.L., Ling, K.V., and Chua, H.C.Low cost SINS/GPS integration for land vehicle navigation.In IEEE $5^{\text {th }}$ International Conference on Intelligent Transportation Systems, Sept. 2002, 910-913.

[8] Jaffe, R., Qi, H., Carter, P., and Madni, A.M.MMQ-G, A low cost MEMS INS-GPS. In ION GNSS $18^{\text {th }}$ International Technical Meeting of the Satellite Division, Long Beach, CA, Sept 13-16, 2005, 956-966.

[9] Lichuan L., Zengshan, T., and Shun-ji, H.An algorithm for integrating GPS/INS attitude determination system In CIE International Conference on Radar, 2001. 167-170.

[10] Qi.H.m, and Moore, J.B.DirectKalman filtering approach for GPS/INS integration IEEE Transactions on Aerospace and Electronic Systems, 38, 2 (Apr. 2002), 687-693.

[11] Agarwal.V.,Arya .H., andBhaktavatsala.S.Design and Development of a Real-time DSP and FPGA-Based Integrated GPS-INS System for Compact and Low Power Applications. In IEEE Transactions on Aerospace and Electronic Systems, 45, 2(Apr.2009) 443-454.

[12] Schmidt,G.T., "Strapdown Inertial Systems-Theory and Applications",AGARD Lecture Series,No.95,1978

[13] Bar-Itzhack,I.Y.,andBerman,N., "Control Theoretic Approach to Inertial Navigation Systems,"Journal of Guidance,Vol.11,No.3,1988,pp.237-245.

[14] ADXL212 Analog Devices, datasheet.http://www.analog.com/UploadedFiles/Data Sheets/accessed 2013.

[15] DXRS652 Analog Devices, datasheet.http://www.analog.com/UploadedFiles/Data Sheets/; accessed 2013.

[16] GPS-Receiver JP3 Falcom Wireless Communication, users manual, www.falcom; accessed 2013.

[17] http://www.Xylinx.com; accessed 2013.

[18] ] http://www.Zedboard.org; accessed 2013.

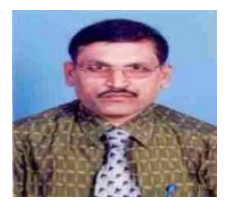

BhogadiLokeswaraRao was born on Jan 4,1968.He received his Bachelor's degree in Electronics \& Communication Engineering fromAndhra University, and Master's degree in Microwave \& Radar Engineering from Osmania University. He is currently an Associate Professor in the Department of Electronics \& Communication Engineering, Bharat Institute of Engineering \& Technology , Hyderabad and is also pursuing Ph.D from Andhra University. He has 24 years of experience in Teaching, R\&D, and Industry. He is working in the area of GPS/INS Integration using Kalman filtering using FPGAs. He is a Fellow of IETE and also a Fellow of IE (India).

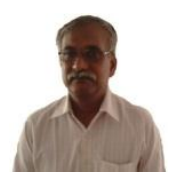

Prof.KVVS Reddy was born on August 14, 1951. He posseses B.E., M.E., and Ph.D degrees. He woked as a Professor in the Department of Electronics \& Communication Engineering of College of Engineering, Andhra University, Visakhapatnam for more than 32 years..He also worked in ECIL, Hyderabad for three years before joining AU. Under his guidance $10 \mathrm{Ph}$.Ds are awarded and few more are pursuing their Ph.Ds. His research interests are in Mobile communication, DSP, Navigation, Radar and Microwave.

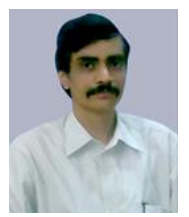

Prof.G.SasibhushanaRao was born on August 15, 1964. He posseses B.E., M.E., Ph.D and MBA(HRD\&Mkting) degrees. He is currently working as Professor \& HOD in the Department of Electronics \& Communication Engineering of College of Engineering, Andhra University, Visakhapatnam. He has 28 years of experience in Teaching, R\&D, and Industry. Under his guidance $7 \mathrm{Ph} . \mathrm{Ds}$ are awarded and few more are pursuing their Ph.Ds.. His research interests are in Navigation, GPS Signal processing, Radar and Microwave. He is a Senior Member of IEEE, USA, Fellow of IETE, Member of International GNSS Society,Australia,,Member of IEEE Comm.Society, USA,Permanent Member of Indian Geophysical Union(IGU),NGRI and Chartered Engineer for IETE .He has published 290 research papers in various International/National Journals/Conferences including IEEE.He is a Reviewer for IEEE, IETE, Indian Academic Journals and other International Journals. He received Dr.SurvepalliRadhakrishnan Award for Best Academician and also Best Researcher Award from Andhra University. He published 3 text books on GPS, Cellular \& Mobile Communications and Electromagnetic Field Theory \& Transmission Lines from Mc-GraHill Publications, New Delhi, Pearson International, New Delhi and John Wiley \& Sons respectively. 